\title{
Diesel engine vibration monitoring based on a statistical model
}

\author{
Suzana Lampreia ${ }^{1, *}$, José Requeijo ${ }^{2}$, and Victor Lobo $^{3}$ \\ ${ }^{1}$ Portuguese Naval Academy, Centro de Investigação Naval (CINAV), Portugal \\ ${ }^{2}$ Faculty of Science and Technology of the Universidade Nova of Lisbon, Mechanical and Industrial \\ Engineering Department \\ ${ }^{3}$ Portuguese Naval Academy, Centro de Investigação Naval (CINAV), Portugal
}

\begin{abstract}
There are various causes for vibrations on diesel engines. The engine vibrations depend not only on the present state of the engine, but also on the fuel quality, the environmental conditions (sea state in the case of shipborne engines), type of casing, other equipment in the vicinity, etc. The engines used for this study are installed aboard a ship, and the main aim is to use modified control charts to assess the condition of the engine and recommend corrective measures when necessary. An important issue to do this correctly is choosing the right places to measure vibration. The collected data is vibration at various engine power levels, measured at various points. The engine's fault history is taken into consideration, but proved to be almost irrelevant. By using modified control charts, the engine vibrations can be estimated, and faults can be detected and classified so as to take corrective actions. In this study we followed a methodology that is slightly different from our previous work, and achieved good results.
\end{abstract}

\section{Introduction}

Vibration measurements can be one of the most important parameters for equipment condition monitoring. The vibration data used in this paper is in the time domain and is measured in three axes. Many factors can influence the diesel engine vibration signature, such as the type of fuel [1], type of mount, other moving components, etc. [2-3]. Abnormal vibrations can also occur due to extreme conditions, such cylinders that are isolated (i.e. are not burning fuel) [4].

Since collecting data is expensive, we must define carefully the methodology to measure only the essential data that allows us to extract the necessary information [5-6]. Various statistical methods can be applied to extract information from data [7]. In this study, we decided to apply modified control charts [8].

\section{Modified control charts on diesel generators monitoring}

\footnotetext{
* Corresponding author: suzanalampreia@gmail.com
} 
Control charts are usually used in process control and use the mean as the reference. If we use the maximum value admissible instead of the mean as reference, we obtain a modified control char that may be used for vibration monitoring of a diesel engine [8].

\section{2. $1 Q$ modified control charts}

For this vibration study we decide to apply the $Q$ modified control chart, which is based on the Quesenberry control charts, and because there is a lack of data, and we cannot control accurately the mean of variable $X$, we transform the data obtaining a $Q$ characteristic. The $Q$ characteristic in instant $r$ is defined as:

$$
Q_{r}\left(X_{r}\right)=\Phi^{-1}\left(G_{r-2}\left(\sqrt{\frac{r-1}{r}}\left(\frac{X_{r}-\left(T_{L}\right)_{r-1}}{S_{r-1}}\right)\right)\right), \quad r=4,5, \ldots
$$

where $X_{r}$ is the observation at instant $r, \Phi^{-1}(\bullet)$ is the Inverse of Normal distribution function, $G_{v}(\bullet)$ is the t-Student distribution function with $v$ degrees of freedom, $\left(\mathrm{T}_{L}\right)_{r-1}$ is the limit value at instant $r-1$, and $S_{r-1}$ is the standard deviation of the observations at instant $r-1$.

In the modified control chart the condition monitoring will start at time $r=4$ [9], and the graph plots $Q_{r}\left(X_{r}\right)$ as a function of $r$. The difference from the traditional process control charts is that the value of $\bar{X}_{r-1}$ is substituted by $\left(\mathrm{T}_{L}\right)_{r-1}$ which represents the limit defined by the manufacturer or International Standards, considering $\left(\mathrm{T}_{L}\right)_{r}=\left(\mathrm{T}_{L}\right)_{N}-3 \sigma_{r-1}$ for observation number $(r-1)$, where $\left(\mathrm{T}_{L}\right)_{N}$ is the standard vibration limit and $\sigma_{\mathrm{r}-1}$ is the standard deviation at instant $r$-1.

The mean and the variance in the instant $r$ are:

$$
\begin{gathered}
\bar{X}_{r}=\frac{1}{r} \sum_{j=1}^{r} X_{j} \\
S_{r}^{2}=\frac{1}{r-1} \sum_{j=1}^{r}\left(X_{j}-\bar{X}_{r}\right)^{2}
\end{gathered}
$$

The mean and the sample variance in the instant $r\left(\bar{X}_{r}\right.$ and $\left.S_{r}^{2}\right)$, can be calculated in function of $X_{r}$ (observation in instant $r$ ) and in function of the values on the instant before $(r-1)$.

As the $Q(X)$ variables are Normal distributed by the standard normal distribution, the limits to the charts, considering only the maximum values, equal or above zero, are given by:

$$
\begin{gathered}
U C L=3 \\
A L=2.5 \\
C L=0
\end{gathered}
$$

considering the vibration limits defined by the equipment manufacturer. The analysis of $Q(X)$ modified charts are not like in the $Q(X)$ chart, so the rules for intervention are different. The data rules analysis are described on the proposed methodology. 


\section{Methodology}

To define the methodology for diesel generator vibration condition monitoring we consider that the equipment is reparable, the data is independent. The proposal sequential actions are:

- Choose the points of measure of diesel generator.

- Collect Vibration Data of the defined 6 points of measure to diesel generator monitoring.

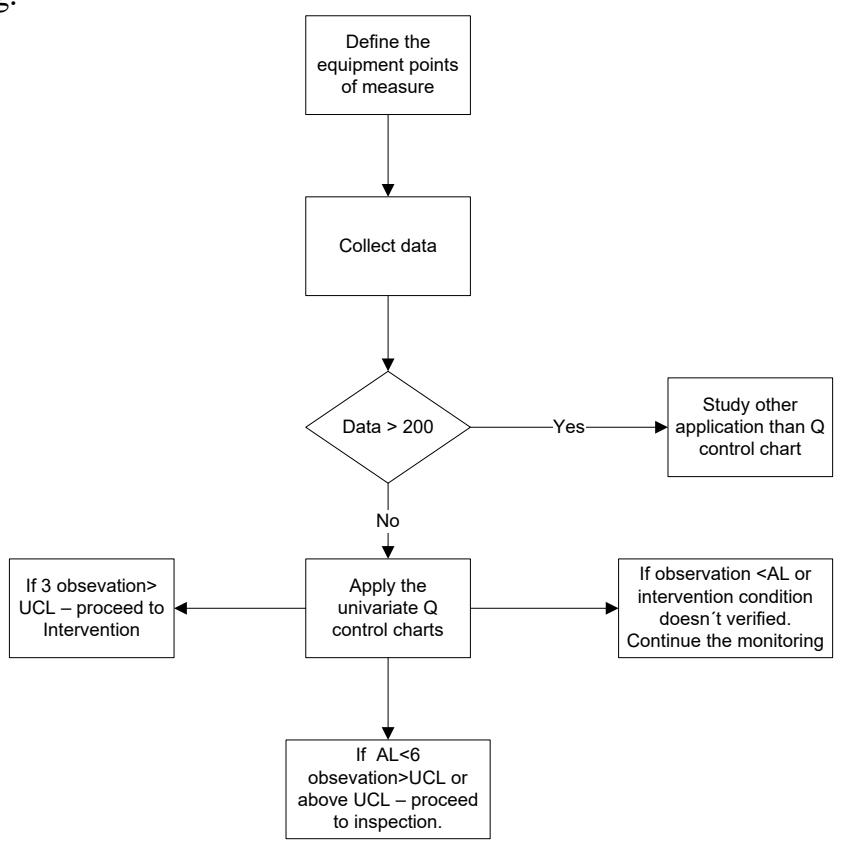

Fig. 1. Diesel generator monitoring methodology.

- The rules of intervention are

- If 3 consecutive observations are above the $U C L$, procced to an intervention maintenance.

- If 6 observations are between $A L$ and the $U C L$, procced to an inspection.

\section{Case study results}

For this study we use two diesel generators, $\mathrm{nr} 1$ and $\mathrm{nr} 2$, and for each selected point the vibration was monitored and analysed, Fig. 2.

With the $Q$ univariate control charts, we observe the six points of vibration measure of the diesel generators.

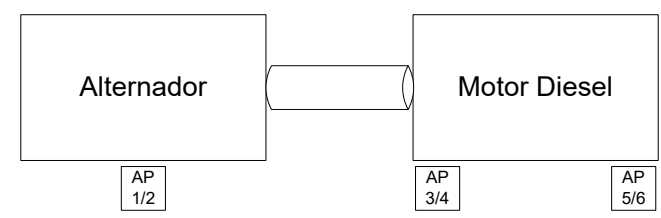

Fig. 2. Diesel generator points of measure. 
In the multivariate $Q$ control charts, we opted to study the points: AP3 V, AP4 V, AP5 $\mathrm{V}$ and AP6 V, because the vibrations on these are higher than in the points AP1 V and AP2 V. We will show only the most representative measures for AP5 and AP6. The data was taken on the three axes, vertical $(\mathrm{V})$, horizontal $(\mathrm{H})$ and axial $(\mathrm{A})$. The vibration data represented in the article is from the vertical measure.

The measures are made annually on the diesel generators. Various measures are taken at each time.

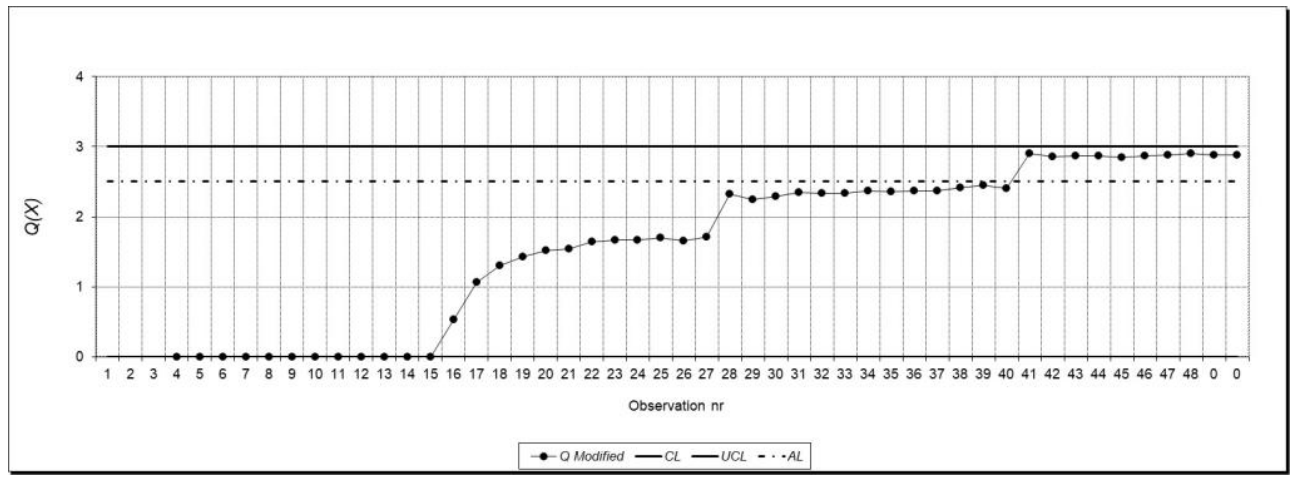

Fig. 3. Diesel generator nr $1-$ AP6.

The representation of Fig. 3 and Fig. 4 shows the increase of the vibration level on each year. On the last year the measure is higher than the fabricant defined limits.

As we can observe for diesel generator $\mathrm{nr} 1$ the vibration increases and stabilizes under the $U C L$, by the defined rules on the observation $\mathrm{nr} 45$ inspection should be made to the diesel generator, considering the use of non-destructive analysis and the observation of another parameters tendency.

For the diesel generator nr 2 the tendency is also to increase, but then it stabilizes. On observation $\mathrm{nr} 33$ we should procced to an intervention.

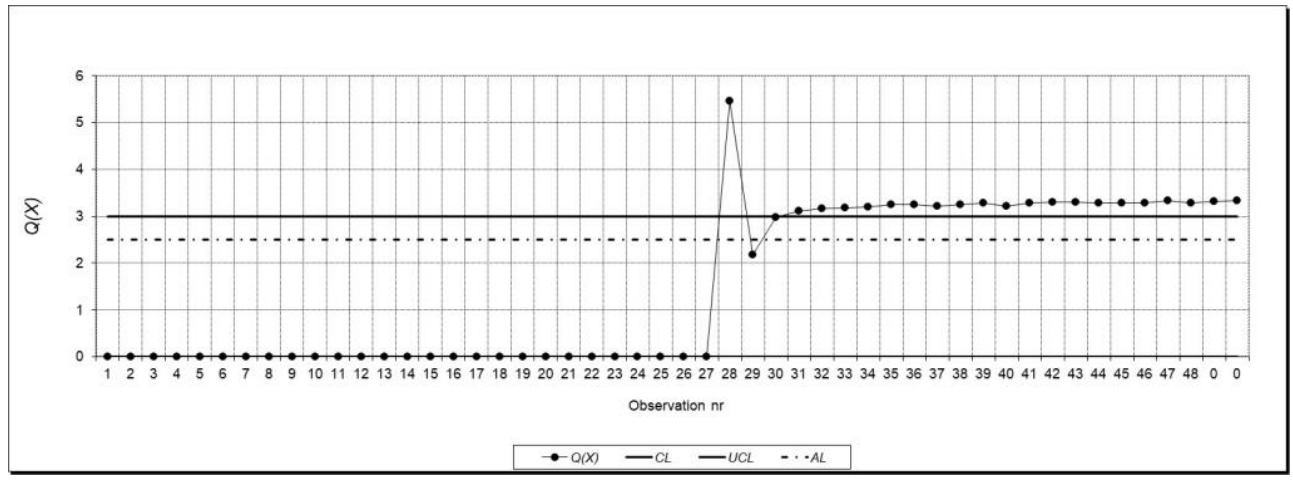

Fig. 4. Diesel generator nr 2 - AP6.

The diesel generator faults along lifecycle was not significant, and its tendency is steady.

To know which anomaly the diesel generator has, the vibration spectrum should be analysed. This study was not in the scope of the actual investigation.

The obtained results confirm a real anomaly on the diesel generators, they both need supports substitutions of the diesel engine. 
When the maintenance technician detects the tendency for vibration increase, they should decrease the interval between measures.

When there is enough data, other charts should be considered for condition monitoring.

\section{Conclusions}

The definition of points of vibration measures are crucial for diesel engine condition monitoring.

The methodology and the define rules identify the need of an inspection and maintenance intervention.

The measures show an anomaly on the diesel engine of the system diesel generator. This was a real anomaly, and after the last measures, the diesel engine supports were substituted.

The application of statistical methods can contribute for an accurate equipment condition control.

The $Q$ modified control charts can be used on vibration condition control on diesel generators systems.

Portuguese Naval School and CINAV are kindly acknowledged for the participation on this conference and also for the fruitful collaboration of the Faculty of Science and Technology from the Universidade Nova of Lisbon.

\section{References}

[1] S. Dehkordi, M. Almassi, A. Borghei, B. Beheshti, Int. J. of Agr. and Cr. Sci., 20842090, (2013)

[2] A.S. Mendes, P.S. Meirelles, D.E. Zampieri, Proceedings of the Institution of Mechanical Engineers, Part K: J. of Multi-body Dyn., 222(2), 155-178 (2008)

[3] R. Amstrong, Fault assessment of a diesel engine using vibration measurements and advanced signal processing, Institutional Archive of the Naval Postgraduate School, (1996)

[4] E. Ftoutou, M. Chouchane, N. Besbès, R. Ouali, Detection of diesel engine misfire by vibration analysis in the time domain, Congrès Tunisien de Mécanique COTUM'08, Hammamet, 17-19 March (2008)

[5] Y. Alhouli, A. Alkhaledi, A. Alzayedi, M. Alardhi, A. Abed, Gl. J. of Res. in Eng.: Gen. Eng., 15(6) Version 1.0, USA (2015)

[6] Z. Li, X. Yan, Z. Guo, Y. Zhang, C. Yuan, Z. Peng, Elec. and Elect. Eng. - Comp. Tech., 109-112 (2012)

[7] D. Watzenig, M. Sommer, G. Steiner, Intech, Diesel Engine - Combustion, Emissions and Condition Monitoring, Chapter 9, 217-230 (2013)

[8] S. Lampreia, J. Requeijo, Mathematical Models and Methods in Modern Science, 200205, Ed. Math. and C. in Sci. and Eng. Ser., Editor: Ana Julia Viamonte, WSEAS, (2012)

[9] Z. Pereira, J. Requeijo, Qualidade: Planeamento e Controlo Estatístico de Processos (Quality: Statistical Process Control and Planning), FCT/UNL Foundation Editor, Lisboa. (2012) 